\title{
Long-term follow-up of a prospective trial of argon laser photocoagulation in the treatment of central serous retinopathy
}

\author{
LINDA FICKER, GILLIAN VAFIDIS, ADRIAN WHILE, AND PETER LEAVER \\ From Moorfields Eye Hospital, City Road, London
}

SUMMARY In a prospective randomised trial of argon laser photocoagulation in the management of central serous retinopathy, long-term follow-up (6.4 to $12 \cdot 1$ years) revealed no evidence that treatment significantly influenced the visual outcome as measured by the Snellen chart and by the Farnsworth-Munsell 100-hue test. Treatment did not reduce either the recurrence rate or the prevalence of chronic disease. Complications of treatment were uncommon. The justification for argon laser photocoagulation appears to be limited to the hastening of symptomatic relief by earlier resolution of serous detachment.

In central serous retinopathy (CSR)' there is serous detachment of the macular retina. It generally follows an acute course, with spontaneous resolution and good recovery of visual function. A chronic course supervenes in a minority of patients, in whom progressive retinal pigment epithelial changes are associated with permanent impairment of visual function. This course may be a consequence of either multiple recurrent attacks or chronic detachment.

The development of suitable photocoagulation techniques ${ }^{2}$ has enabled accurate treatment to the source of subretinal fluid as determined by fluorescein angiography. ${ }^{34}$ Acute studies have shown that the duration of macular detachment is reduced by focal application of argon laser photocoagulation to the sites of 'leakage' in the retinal pigment epithelium. ${ }^{5-8}$ Resolution of serous detachment is associated with symptomatic improvement following photocoagulation, ${ }^{79-11}$ though it is acknowledged that symptoms may persist to a variable degree despite reattachment of the retina. ${ }^{412}$

Sixty-seven eyes in a randomised controlled clinical trial, were initially reported on in $1979 . .^{5}$ In the original study there was no significant difference in final visual outcome between the treated and untreated groups of patients as judged by Snellen acuities and Farnsworth-Munsell 100-hue discrimination. No patient suffered visual loss because treatment was withheld. The purpose of our long-term

Correspondence to L A Ficker, FRCS, Professorial Unit, Moorfields Eye Hospital, City Road, London EC1V 2PD. study was to establish the recurrence rate of CSR in treated and untreated patients. In addition we wished to identify any differences in long-term visual outcome between the two groups and any complications of treatment, factors which are important in a recurrent disease affecting the socioeconomic potential of young people.

\section{Patients and methods}

Seventy consecutive eyes with CSR in 69 patients referred to the Retinal Diagnostic Department of Moorfields Eye Hospital between 1973 and 1977 satisfied the following criteria for admission to the study: (1) corrected visual acuity (VA) of $6 / 12$ or better; (2) retina detached at the fovea; (3) retinal pigment epithelial (RPE) defects smaller than one disc diameter (DD); (4) no symptomatic improvement since the onset of the attack; (5) absence of subretinal exudates; (6) no cystic retinal oedema present; (7) no associated ocular disease; (8) informed consent to participate in the study. Patients were selected at random by drawing a card in a sealed envelope so that half were treated and half untreated. It was ruled that eyes in the untreated group in which the corrected VA fell to less than 6/12 during the study or which developed cystic retinal oedema or subretinal exudates should be treated and classified as cases of failed conservative management. No patient was withdrawn from the study for these reasons. 
Fluorescein angiography was used to identify the leaking site, which was then treated with the Coherent Radiation 800 argon laser using burns of 50 to $200 \mu \mathrm{m}$ in diameter, 20 to 200 milliseconds in duration, and 150 to 400 milliwatts of power. Initial and final examinations included clinical history, assessment of best corrected VA, Farnsworth-Munsell 100-hue error score, funduscopy by both the Hruby lens and the indirect ophthalmoscope, and fluorescein angiography.

Of the 70 eyes entered into the study three were excluded from the original report because they had not been followed up for six months. A further four had missed visits. Hence data from 63 eyes were initially reported. Of the 70 eyes $44(63 \%)$ were available for review at intervals ranging from 6.4 to $12 \cdot 1$ years after their initial presentation. Changes in patients' domicile and social status made contact difficult, while some patients, particularly those who had not received treatment, were reluctant to spend time attending for review. Twenty-one patients could not be traced, two had died, two refused to attend, and one was known to have emigrated.

The mean follow-up for the untreated group $(n=19)$ was 9.5 years and for the treated group $(n=25) 9 \cdot 1$ years. In the untreated group six patients were treated for recurrence after the initial attack and were reclassified as a separate group of subsequently treated patients (group S). The remaining 13 untreated patients were designated group $U$ and the 25 treated patients group $T$.

\section{Results}

Persistent symptoms (mostly paracentral scotomata, minimal distortion, and colour desaturation) were reported by 23 patients; $6(47 \%)$ in group $U, 5(83 \%)$ in group S, and $12(48 \%)$ in group T. Visual acuities among these patients were $6 / 9$ or better, with the exception of one patient with extensive disease whose VA fell to 6/60 in group S (Fig. 1). The scores for visual acuity and 100-hue discrimination for this patient were excluded to allow statistical analysis.

\section{VISUAL ACUITIES}

Visual acuities were converted to a simple numerical scale from $1(6 / 4)$ to $5(6 / 12)$. The mean VA on presentation, as originally reported, was 3.9 in the control group ( 3.8 in group $U$ and 4.2 in group $S$ ) and 3.7 in the treatment group. At final follow-up the visual acuities for the three groups were 2.0 (group $\mathrm{U}$ ), 2.0 (group $\mathrm{S}$, excluding the only patient in the study to fall below $6 / 12$ to $6 / 60$ ), and 1.9 (group T). Visual acuities of $6 / 6$ or better were achieved in 37 patients of the 44 at long-term follow-up; $12(92 \%)$ in group U, $5(83 \%)$ in group S, and $20(80 \%)$ in group
T. There were no significant differences in VA between untreated patients (group U) and patients randomised to treatment (group T), either initially $(p=0.606)$ or at final follow-up $(p=0.489)$ (MannWhitney U test).

\section{HUE DISCRIMINATION}

Results were available for 35 cases. The mean error scores at presentation were 243 in group U, 284 in group S, and 290 in group T. The final error scores were 104 in group U, 99 in group S (excluding the patient with final VA $6 / 60$ whose score was 787 ), and 112 in group T. These represent changes of $59 \%$, $70 \%$, and $60 \%$ respectively. There were no statistically significant differences between the error scores for untreated patients and those randomised to treatment either initially $(\mathrm{p}=\mathbf{0 \cdot 6 7 )}$ or at final followup $(p=0.94)$.

\section{RECURRENCES}

Thirteen patients gave histories of recurrence during the follow-up period $-37 \%$ of the original control group and $28 \%$ of the treatment group. The difference was not statistically significant $(p=0 \cdot 76)$. A further eight were found to have fluorescein angiographic appearances consistent with recurrent disease. Hence angiographically documented recurrence occurred in $53 \%$ of the original control group and $44 \%$ of the treatment group. This difference was not significant $(p=0.79)$.

Of the 13 symptomatic patients five presented at the time of their recurrence. The site of recurrence could therefore be identified in these cases. Two patients (group U) presented within 12 months of the original episode and three presented later, between 15 and 29 months (two treated patients and one control). The recurrences were multifocal and included the original focus of leakage (Fig. 2) in the untreated patients. Recurrence occurred within 200 $\mu \mathrm{m}$ of the original leak, on the border of treated pigment epithelium, in treated patients. One treated patient had a new remote site of leakage in addition to two new leaks adjacent to the original foci (Fig. 3).

The chronic phase of the disease represents a threat of permanent visual loss and is characterised by progressive retinal pigment epithelial disturbance. Of the 41 patients on whom follow-up fluorescein angiography was performed two untreated, five treated patients, and two from group $\mathrm{S}$ were found to have diffuse hyperfluorescence exceeding $3000 \mu \mathrm{m}$ (2 DD). Of these, eight gave no history of recurrence during the period of progressive retinal pigment epithelial change. The distribution of this change was inferior to the original sites of documented leakage in each case. There was no difference in the prevalence of chronic disease between the groups. 


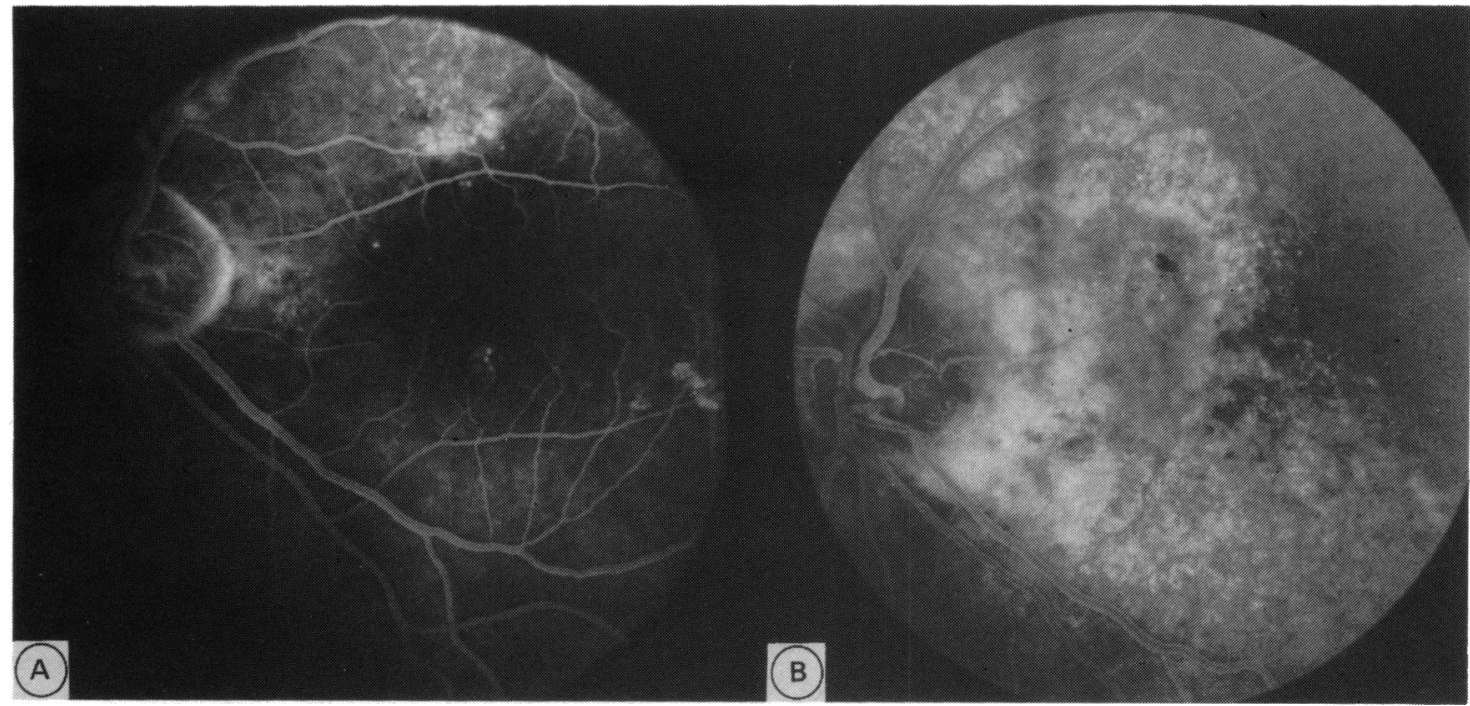

Fig. 1 A: Resolution of CSR following treated recurrence. B: Progressive RPE disturbance eight years after treatment.

\section{COMPLICATIONS OF TREATMENT}

Choroidal neovascularisation was identified at the sites of treated focal leakage in two patients, but neither was symptomatic (Fig. 4). In both cases the treatment site was within $750 \mu \mathrm{m}$ of the fovea. A further patient showed angiographic evidence of leakage without a clearly identifiable capillary tuft at the photocoagulation site; choroidal neovascularisation could not be excluded as the cause. The neovascular membranes did not involve the fovea at follow-up intervals between eight and nine years after treatment, and VAs in these three eyes were $6 / 5$ or better.

\section{Discussion}

The main issue we are concerned to address is the role of photocoagulation in the management of central serous retinopathy. We defined our diagnostic criteria and selected patients with good initial visual acuity for whom a decision concerning treatment presents a dilemma given the expected spontaneous resolution of the disease.

The long-term visual outcome in our study was good and was not significantly different in treated patients as compared with untreated patients. A similar result was observed in a randomised trial of

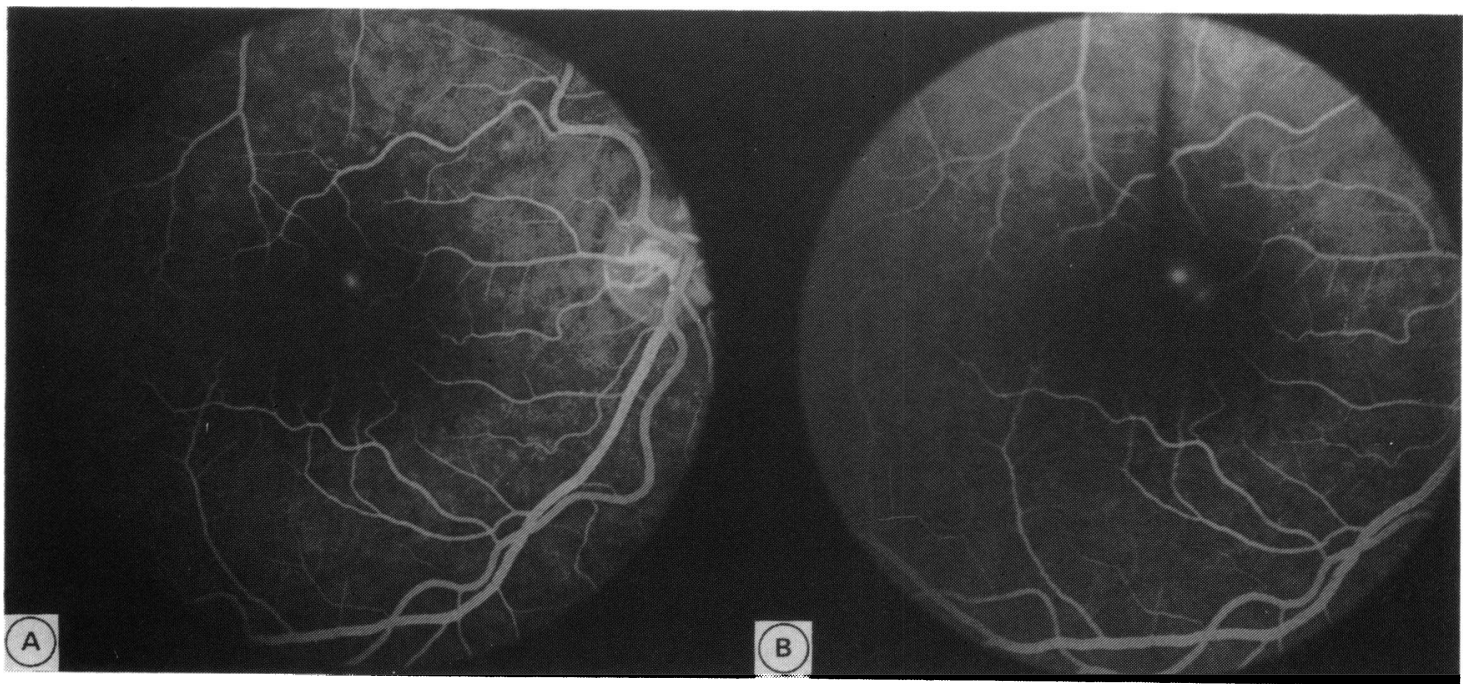

Fig. 2. A: Leakage in acute CSR. B: Multifocal recurrence including original focus. 


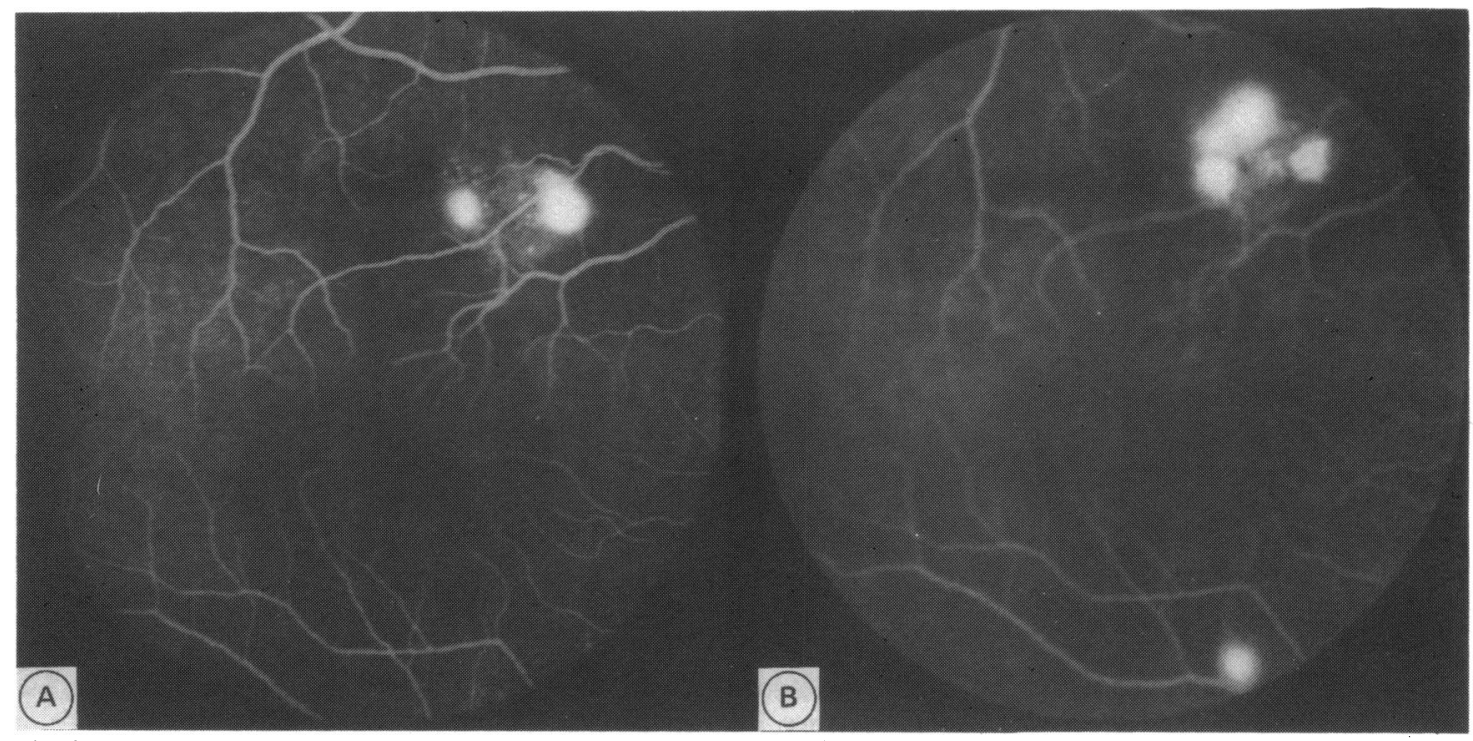

Fig. 3 A: Leakage in acute CSR. B: Recurrence adjacent to treated sites.

treatment by Watzke et al., ${ }^{7}$ while Klein et al. ${ }^{13}$ reported spontaneous recovery with vision of $6 / 12$ in 27 consecutive patients treated conservatively.

The rationale for treatment as postulated by Maumenee $^{3}$ was that central serous retinopathy resulted from leaks through defects in Bruch's membrane and the adjacent retinal pigment epithelium and that focal photocoagulation would occlude these defects.

While it is true that direct photocoagulation reduces the duration of detachment in comparison with indirect or no treatment, ${ }^{814}$ the mechanism by which this is achieved remains controversial. Spitznas ${ }^{15}$ has reviewed the experimental evidence on the nature of the retinal pigment epithelial abnormality. He postulated a reversal in the direction of water transport across the abnormal retinal pigment epithelium. such that this occurs from the choroid towards the neuroretina. ${ }^{16}$ Photocoagulation may achieve resolution of detachment by destroying the abnormal focus of pigment epithelial cells and inducing migration of adjacent epithelial cells across the treatment site, hence restoring function to the focus of 'leakage'. In our study recurrence did not occur at

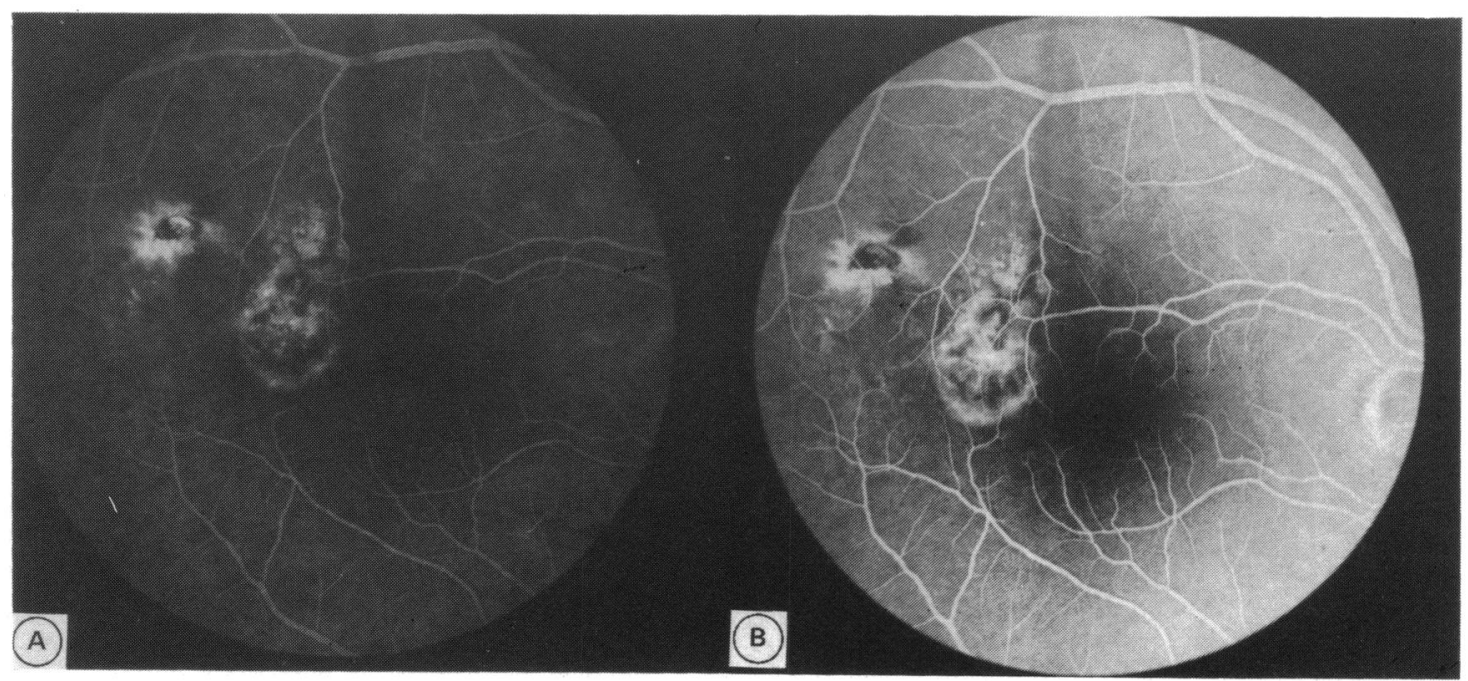

Fig. 4 A, B: Choroidal neovascularisation at treatment site. 
the treatment site but from a focus about $200 \mu \mathrm{m}$ from it, while recurrence in untreated patients did indeed occur at the site of the original leak. There was, however, no difference in the recurrence rate of acute CSR between the two groups.

The recurrence rate among treated and untreated patients has not hitherto been established for a prospective, randomised trial, and existing data are not concordant. No significant difference was found by Watzke et al. ${ }^{14}$ in a prospective study, nor by Nanjiani ${ }^{11}$ or Gilbert et al. ${ }^{17}$ in retrospective studies, while Dellaporta ${ }^{18}$ and Robertson and Ilstrup ${ }^{8}$ believed there was a reduced incidence of recurrence among treated patients. Robertson and Ilstrup ${ }^{8}$ found no recurrence among a small number $(n=7)$ of treated patients whose outcome was reported at 18month follow-up. In our long-term prospective study we found no evidence that treatment reduced the recurrence rate of acute CSR.

Of particular interest was the finding that treatment did not reduce the incidence of chronic disease. The concept of chronic disease is now widely accepted, though it is uncertain whether this represents recurrence or the chronic passage of fluid across the retinal pigment epithelium (RPE) towards the neuroretina. ${ }^{78-21}$ The manifestation of chronic disease is the involvement of large areas of abnormal RPE, contiguous with the original site of leakage and often gravity-dependent, which are angiographically evident as areas of hyperfluorescence at the level of the RPE. Our results do not support the concept that chronic disease results from recurrence, since only one patient with chronic disease had a history of recurrent disease and of the 13 with symptoms of recurrent attacks only one had chronic disease.

The most serious complication of treatment is choroidal (subretinal) neovascularisation as reported by Schatz et al..${ }^{22}$ It is assumed that, when a diagnosis of CSR is made, early neovascular membranes (NVM) can be specifically excluded as a cause of serous detachment. In earlier series this possibility was not fully appreciated, and of the 27 cases reviewed by Schatz et al. a proportion did indeed have NVMs rather than CSR at the time of diagnosis. In others NVMs were suspected but not proved. Of the cases of true CSR in which development of an NVM followed laser treatment, the common factor was the proximity of the leakage site to the fovea. Schatz et al. postulated that the combination of highpower densities delivered and the high concentration of absorbent pigments at the fovea probably resulted in ruptures of Bruch's membrane, hence stimulating neovascularisation. These cases were symptomatic and therefore presented to an ophthalmologist. NVMs have not been reported in many series. Watzke et al. ${ }^{14}$ reported two among 20 treated cases and these involved central vision. Our cases, however, demonstrate that NVMs distant from central vision may be both asymptomatic and stable, which may account for the paucity of NVMs complicating treatment which have been reported, particularly in retrospective studies.

Most patients with CSR who present with VAs equal to or better than $6 / 12$ achieve recovery of good visual acuity, which is hastened by laser treatment. The only patient to suffer visual deterioration was originally in the untreated group. The visual outcome, however, as measured by Snellen acuities and Farnsworth Munsell 100-hue discrimination, does not necessarily measure all attributes of visual function and permanent symptoms existed in patients with good recovery of function as measured by these techniques. It was found by Chuang et al. ${ }^{23}$ that rod threshold elevation during acute CSR exceeded 3.5 log units and was directly related to the height of the detached retina. Recovery of threshold to within 0.5 log unit of normal was recorded within three weeks of clinical resolution. Thus a small long-term deficit remained. Chronic disease was characterised by threshold elevation of $3.5 \mathrm{log}$ units in areas of abnormal RPE in the absence of detectable neurosensory detachment.

There was incomplete ascertainment, since only $63 \%$ of the patients entered into the trial were reviewed, and it is possible the results may be biased. This is unlikely, because the main reason for failure at follow-up was that patients were untraceable, and this would not be expected to be biased by visual outcome.

We conclude that treatment does not reduce the incidence of recurrent disease or of chronic CSR in which progressive retinal pigment epithelial disturbance presents a real threat of permanent visual loss. Our results suggest that the conclusions drawn from the original study remain valid; that the role of argon laser photocoagulation in CSR with good visual acuity is limited to hastening relief of symptoms by achieving speedier resolution of serous detachment.

We thank Professor Alan Bird for his help and encouragement in the preparation of this manuscript, Peter Clark for the statistical evaluation of our data and the Department of Medical Illustration, Moorfields Eye Hospital, for preparation of the photographs. The study was supported by the Locally Organised Research Scheme, Moorfields Eye Hospital.

\section{References}

1 von Graefe A. Ueber central recidivirende Retinits. Graefes Arch Clin Exp Ophthalmol 1866; 12: 211-5.

2 Wessing A. Central serous retinopathy and related lesions. In: Horatio Ferrer Eye Institute Publications Committee, ed. Modern problems in ophthalmology. Proceedings of the International Symposium on Fluorescein Angiography, Miami, Floria. Basel: Karger, 1971; 9: 148-51. 
3 Maumenee AE. Symposium: macular diseases, clinical manifestations. Ophthalmology 1965; 69: 605-13.

4 Gass JDM. Pathogenesis of disciform detachment of the neuroepithelium. II. Idiopathic central serous choroidopathy. Am J Ophthalmol 1967; 63: 587-615.

5 Leaver $P$, Williams $C$. Argon laser photocoagulation in the treatment of central serous retinopathy. Br J Ophthalmol 1979; 63: 674-7.

6 Burton TC. Central serous retinopathy. In: Blodi FC, ed. Current concepts in ophthalmology. St Louis: Mosby, 1972; 3: 128.

7 Watzke RC, Burton TC, Leaverton PE. Ruby laser photocoagulation therapy of central serous retinopathy. Ophthalmology 1974; 78: 205-11.

8 Robertson D, Ilstrup D. Direct, indirect and sham laser photocoagulation in the management of central serous retinopathy. Am J Ophthalmol 1983; 95: 457-66.

9 Spalter HF. Photocoagulation of central serous retinopathy. Arch Ophthalmol 1968; 79: 247-53.

10 Peabody RR, Zweng HC, Little HL. Treatment of persistent central serous retinopathy. Arch Ophthalmol 1968; 79: 166-9.

11 Nanjiani M. Longterm follow-up of central serous retinopathy. Trans Ophthalmol Soc UK 1977; 97: 656-61.

12 Zweng HC. Discussion of central serous retinopathy. Mod Probl Ophthalmol 1974; 12: 363.

13 Klein ML, van Buskirk EM, Friedman E, Gragoudas E, Chandra S. Experience with nontreatment of central serous choroidopathy. Arch Ophthalmol 1974; 91: 247-50.
14 Watzke RC, Burton TC, Woolson RF. Direct and indirect photocoagulation of central serous retinopathy. $A m J$ Ophthalmol 1979; 88: 914-8.

15 Spitznas M. Pathogenesis of central serous retinopathy: a new working hypothesis. Graefes Arch Clin Exp Ophthalmol 1986; 224: 321-4.

16 Miller S, Farber DB. Cyclic AMP modulation of ion transport across frog retinal pigment epithelium. Measurements in the short circuit state. J Gen Physiol 1984; 83: 853-74.

17 Gilbert CM, Owens SL, Smith PD, Fine SL. Long-term followup of central serous chorioretinopathy. Br J Ophthalmol 1984; 68: 815-20.

18 Dellaporta A. Central serous retinopathy. Trans Am Ophthalmol Soc 1976; 74: 144-51.

19 Wessing A. Changing concept of central serous retinopathy and its treatment. Ophthalmology 1973; 77: OP275-80.

20 Bennett G. Central serous retinopathy. Br J Ophthalmol 1955; 39: 605-18.

21 Jalkh AE, Jabbour N, Avila MP, Trempe CL, Schepens CL. Retinal pigment epithelium decompensation. I. Clinical features and natural course. Ophthalmology 1984; 91: 1544-8.

22 Schatz H, Yannuzzi LA, Gitter KA. Subretinal neovascularisation following argon laser photocoagulation treatment for central serous retinopathy: complication or misdiagnosis? Ophthalmology 1977; 83: 893-906.

23 Chuang EL, Sharp DM, Fitzke FW, Kemp CM, Holden AL, Bird AC. Retinal dysfunction in central serous retinopathy. Eye 1987; 1: 120-5.

Accepted for publication 18 August 1987. 